\title{
Angst vor Infektionen - Angst vor dem Impfen
}

\author{
Cornelia Betsch $^{(\bowtie)}$ \\ Heisenbergprofessur für Gesundheitskommunikation, Psychology and \\ Infectious Diseases (PIDI) Lab, Universität Erfurt, Erfurt, Deutschland \\ cornelia.betsch@uni-erfurt.de
}

Schlüsselwörter: Impfbereitschaft · Akzeptanz $\cdot$ Risikokommunikation · COVID-19 · Coronavirus

\section{Der COVID-19 Snapshot Monitor (COSMO)}

Man sagt, dass die Impfung ihr eigener Feind sei: Der Erfolg von Impfungen führt allgemein dazu, dass Infektionskrankheiten weniger sichtbar sind und wir meist gar nicht mehr verstehen, warum überhaupt geimpft werden soll [1]. Es stellt sich gerade die Frage: Ändert sich das mit COVID-19? Wir stehen an einem sehr spannenden Punkt, denn wir können jetzt für eine ganz neue Impfung beobachten, wie Impfmüdigkeit eigentlich entsteht. Seit die ersten rund 100 Fälle in Deutschland im März aufgetreten sind, gibt das Team vom COVID-19 Snapshot Monitor (COSMO) [2, 3] wöchentlich oder zweiwöchentlich - je nach Infektionslage - Einblicke in die psychologische COVID-19-Lage: Was denken die Menschen über Corona? Was fühlen sie? Wie schützen sie sich? Wie hängt das alles miteinander zusammen? Dieser Einblick in die psychologische Lage soll hier kurz vorgestellt werden, denn daraus lässt sich viel über das Impfen allgemein, über Risikowahrnehmung und über grundlegende Zusammenhänge lernen.

\section{Erwartungen zur, ,zweiten Welle“6}

Das COSMO-Team hat im März, im Mai und Ende September die Menschen gefragt, wann sie mit einer zweiten Welle rechnen. Durchweg lautete die Antwort: in zwei Monaten. Als es im September gefragt hat, wurden in Deutschland täglich ungefähr 1000 Neuinfektionen verzeichnet. Jetzt, Ende Oktober 2020, meldet das RobertKoch-Institut täglich rund mehr als 18.000 Neuinfizierte: Die Zahlen steigen also rasant. Die Menschen erwarten auch, dass die Pandemie weitergeht: Etwa $30 \%$ der Befragten gehen davon aus, dass die Pandemie noch 18 bis 24 Monate dauert. Die Awareness, das Bewusstsein, in der Bevölkerung für die Krisensituation ist also hoch. Entsprechend werden auch Nachrichten gut verfolgt. $80 \%$ der Befragten wissen, dass aktuell weder Medikamente noch Impfstoffe verfügbar sind. Die Menschen nehmen das Wissen aus den Medien auf: Wird zum Beispiel über einen russischen Impfstoff 
berichtet, stellt sich die Wahrnehmung ein, dass ein Impfstoff möglich ist, wenngleich die Effekte relativ kurzfristig sind. Ganz grundsätzlich ist es den Menschen bewusst, dass im Moment guter Rat noch teuer ist.

\section{Risikowahrnehmung}

Die Risikowahrnehmung ist ein sehr wichtiger Parameter für Schutzverhalten [4]. Angst, Sorge und beständige Gedanken an Corona sind Variablen, die wir zum affektiven Risiko zusammenfassen. Wenn wir kein Risiko wahrnehmen, dann schützen wir uns auch nicht.

Als das Virus neu war und zudem unbekannt war, wie man sich eigentlich schützen kann, stieg die affektive Risikowahrnehmung schnell stark an. Dies ging mit den damals schnell getroffenen stark restriktiven Maßnahmen einher. Ab Mitte April sank die Risikowahrnehmung. Im August gab es noch einmal ein kurzes Aufbäumen der Risikowahrnehmung als wieder ein leichter Anstieg der Infektionszahlen zu verzeichnen war. Dann passierte in der Politik nichts - und man gewöhnte sich letztlich an den dauerhaften Anstieg der Infektionszahlen. Ende Oktober gab es wieder einen leichten Anstieg der Risikowahrnehmung, der aber viel schwächer ausfiel als der Anstieg der Infektionszahlen. Die Funktion zwischen Reiz und Reaktion schwächt sich also ab: Das kann man als Pandemiemüdigkeit werten. Der Reiz sind hier die Fallzahlen und die Reaktion ist das Schutzverhalten. Dieser Zusammenhang lässt sich auch in Simulationen erkennen. Wird in Simulationen so etwas wie Pandemiemüdigkeit hineinmodelliert, dann tritt klar zutage, dass eine zweite Welle kommen wird.

\section{Impfungen}

Angst vor Infektionen und Angst vor dem Impfen stehen in einem spannenden Zusammenhang. Am Anfang der Pandemie hatten alle Angst vor Corona und dies bewirkte, dass Menschen - unter anderem - nicht mehr zum Impfen gegangen sind. Viele Routineuntersuchungen wurden abgesagt, Operationen verschoben und auch bei sonstigen Beschwerden gingen die Menschen nicht so schnell zum Arzt. Auch Impfungen fanden nicht so häufig wie sonst statt. Und tatsächlich spielte hier die Angst vor dem Impfen auch eine Rolle: Menschen mit wenig Vertrauen in Impfungen haben vereinbarte Impftermine eher abgesagt als Menschen mit hohem Vertrauen [5].

Das zeigt: Gerade jetzt während der Pandemie wird es sehr wichtig sein, dass Wissenschaft und Politik nicht nur auf die COVID-19-Impfung fokussieren, sondern auch auf die bereits existierenden Impfungen, die die Menschen nun möglicherweise auslassen. Man sollte diesen Spillover-Effekt im Blick haben. Denn es gilt das zu schützen, was es an gut funktionierenden Impfprogrammen gibt und was an Impfstoffen verfügbar ist, von denen wir sicher wissen, wie sie wirken, wie sicher sie sind und wer sie wann erhalten soll. 
Man könnte die Covid-19-Pandemie für eine perfekte Bühne für einen Impfstoff halten: Die Bevölkerung ist sich der Naturkatastrophe und deren potenzieller Dauer bewusst und weiß, dass Impfungen im Prinzip der einzige Ausweg sind. Die Impfungen werden auch in den Medien sehr stark herbeigewünscht. Man hofft darauf, dass die Impfung uns erlöst. Die Risikowahrnehmung folgt den Fallzahlen nicht eins zu eins, steigt im Moment aber wieder an. Bekannt ist zudem, dass bei einer ungefähren Basisreproduktionsrate von drei sich wohl etwa $67 \%$ impfen lassen müssten, um das Infektionsgeschehen einigermaßen einzufangen.

Impfbereitschaft. Eigentlich wäre das also eine perfekte Bühne, aber eben nur eigentlich: Bereits im April haben Datenerhebungen einer Hamburger Arbeitsgruppe gezeigt, dass europaweit nur eine mittelmäßige Bereitschaft zur COVID-19 Impfung besteht [6]. In stärker von der Pandemie beeinträchtigten Regionen, wie Norditalien oder auch England, ist die Impfbereitschaft etwas höher, aber vor allem in Deutschland oder Frankreich, die für Impfskeptizismus bekannt sind, ist die Bereitschaft eher mittelmäßig [6].

Die Impfabsicht und die Überzeugung, dass eine Impfung gegen

das Coronavirus verpflichtend sein sollte

Bewertet auf einer Skala von 1 (Ablehnung) bis 7 (Zustimmung).

Mittelwerte und $95 \%$ Konfidenzintervalle.

Prozentangaben geben den Anteil an Personen wieder, die den Aussagen (sehr) zustimmen.

Zwischen dem 19.05 und 09.06. wurde keine Zustimmung zur Impfpflicht erfasst

$6.79 \%$

$79 \%$

6

त. $67 \%$

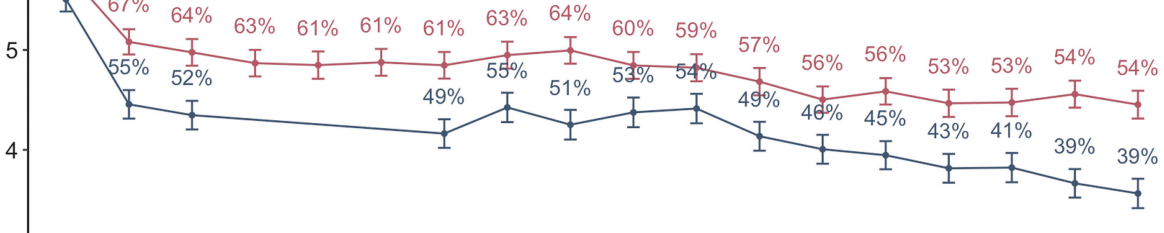

3

2

\begin{tabular}{|c|c|c|c|c|c|c|c|c|c|c|c|c|c|c|c|c|c|}
\hline ষ্ঠ & 10 & மீ) & மீ) & மீ) & ம் & ம் & 今̊ & 今 & $\stackrel{\circ}{0}^{\circ}$ & $\stackrel{\circ}{0}^{\circ}$ & ஓं & த் & ஓं & 음 & $\stackrel{\circ}{\circ}$ & $\check{\check{E}}$ & 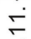 \\
\hline$\underset{\leftarrow}{\square}$ & 10 & $\stackrel{\sim}{\sim}$ & $\stackrel{\circ}{\circ}$ & $\stackrel{\leftrightarrow}{\sim}$ & ஓं & $\ddot{\sim}$ & No & $\bar{\sim}$ & ¿ & $\stackrel{\infty}{\sim}$ & ర & $\stackrel{0}{\leftarrow}$ & ஓి & $\ddot{m}$ & 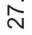 & $\stackrel{\circ}{\circ}$ & $\approx$ \\
\hline
\end{tabular}

$\rightarrow$ Impfabsicht $\rightarrow$ Impfpflicht

Abb. 1. Impfbereitschaft und Zustimmung zu einer Impfpflicht gegen COVID-19 laut Daten des COVID-19 Snapshot Monitorings [3] 
Die Impfbereitschaft in Deutschland lag laut der COSMO-Befragung im Mai noch bei über $60 \%$, aktuell bei rund $50 \%$ (Abb. 1). Selbst, wenn ein effizienter, sicherer Impfstoff bereitstünde, wäre angesichts dieser Impfbereitschaft kein ausreichender Schutz herzustellen - und dies trotz wieder ansteigender Zahlen. Die Impfbereitschaft der Menschen hat also offensichtlich nichts mit der aktuellen Bedrohungslage zu tun. Noch weniger positiv ausgeprägt ist die Zustimmung zu einer Impfpflicht, dargestellt durch die blaue Linie. Wir fragen das dauerhaft ab, um die Policy-Präferenzen der Bevölkerung einschätzen zu können. Die Zustimmung zu einer Impfpflicht ist demnach eher mittelmäßig. Eine Impfplicht wurde durch das Bundesgesundheitsministerium auch wiederholt ausgeschlossen.

Gründe für Impfen oder Nichtimpfen. Im Vergleich zu anderen Impfungen [7], die schon länger auf dem Markt sind, etwa die gegen Masern, ist das Informationsbedürfnis für die Impfung gegen COVID-19 besonders hoch: Wenn man sich impfen lassen möchte, will man gut über Kosten und Nutzen Bescheid wissen.

Momentan nimmt das Vertrauen in Impfungen leicht ab. Vertrauen in die Impfung ist der bedeutendste Faktor - und das stabil über die Zeit. Mit dieser Dauererhebung können wir Impfmüdigkeit beim Entstehen beobachten. In Abhängigkeit von welchen Ereignissen verändert sich die Impfbereitschaft und die Bedeutsamkeit der Einflussfaktoren? Im Moment ist die Sicherheit ein sehr wichtiger Faktor. Zudem spielt die Risikowahrnehmung (complacency) eine Rolle: Ist die Krankheit so bedrohlich, dass eine Impfung in Betracht gezogen wird? Zwar würde die Entwicklung angesichts weiter steigender Fallzahlen sicherlich aufschlussreich sein, aber ich hoffe natürlich, dass wir das nicht erleben müssen, um eine Steigerung der Impfbereitschaft zu erleben.

Die kollektive Verantwortung (collective responsibility) spielt auch eine Rolle: Es ist wichtig, andere zu schützen und von den anderen auch diese Einstellung erwarten zu können [8, 9]. Menschen haben im Laufe der Pandemie sehr viel über Herdenimmunität und Gemeinschaftsschutz gelernt und wissen, dass andere, die immun sind, sie mitschützen können. Das ist eine sehr wesentliche Information, die sich aber auch hinderlich auf die Impfbereitschaft auswirken kann, wenn sich Unentschlossene nicht selbst impfen lassen wollen und sich - vielleicht auch nur zunächst - auf den Schutz der anderen verlassen. Dieser Herausforderung ist auf jeden Fall zu begegnen.

Nicht nur das Muster der relevanten Einflussfaktoren, sondern auch die Impfbereitschaft insgesamt ähnelt aktuell dem Muster der Grippe. Dies ist ein bedeutender Aspekt für unser weiteres Nachdenken über die Impfbereitschaft. Die Risikogruppen sind für Grippe und COVID-19 sehr ähnlich. Wichtig ist der Befund, dass bei COVID-19, wie bei der Grippe, Personen in Gesundheitsberufen derzeit eine etwas geringere Impfbereitschaft haben [3]. Eine Herausforderung bei der Einführung des Impfstoffs wird daher darin bestehen, eine hohe Impfrate in dieser Gruppe zu erreichen, die die Impfung wahrscheinlich als Erstes bekommen wird. Aus der Forschung zur Grippeimpfung ist bekannt, dass die individuelle Einstellung zum Impfen eine Rolle spielt: Wer selbst gut geimpft ist, empfiehlt die Impfung auch häufiger [10]. Die Impfung wird von Ärzten seltener empfohlen, wenn sie sie für weniger effektiv halten, wenn sie die Nutzen-Risiko-Relation der Impfung als schlecht bewerten oder Angst vor Nebenwirkungen haben. Über diese Gesichtspunkte wissen wir hinsichtlich COVID-19-Impfungen zwar momentan recht wenig 
und wesentliche Informationen dazu werden möglicherweise erst im Laufe der Zeit bekannt. Doch aus dem Empfehlungsverhalten der Ärzte bei den Influenza-Impfungen [10] lässt sich jetzt schon vorsichtig schließen, dass es angesichts der Unsicherheiten bei der COVID-19-Impfung Probleme bei der Impfbereitschaft bei den Menschen in Gesundheitsberufen geben wird.

Eine weitere Herausforderung besteht darin, dass einige ganz neue Impfstofftypen zum Teil beim Menschen noch gar nicht zugelassen sind. Das betrifft nicht nur den mRNA-Impfstoff. Auch Vektor-Impfstoffe gibt es erst relativ wenige. Eine gesellschaftliche Diskussion nimmt langsam Gestalt an, beispielsweise stellen große Zeitschriften und Zeitungen diese Impfstofftypen vor. Dass die Entwicklung mehr oder weniger mit Gentechnik in Zusammenhang steht, ist noch keine weit verbreitete Erkenntnis. Möglicherweise könnte dies in der Kommunikation und für die Akzeptanz schwierig werden, besonders wenn dies durch kleine Gruppen von Impfgegnern ausgeschlachtet wird. In jedem Fall ist hierauf frühzeitig ein Augenmerk zu richten.

Innovative Technologien sorgen dafür, dass wir vermutlich sehr schnell über Impfstoff in ausreichenden Mengen verfügen werden. Das ist den Menschen wichtig, doch bedeutender ist das Vertrauen, dass keine Nebenwirkungen auftreten, aber langfristiger Schutz und hohe Effektivität bestehen [3]. Letztlich gilt es, ein Bewusstsein für die Vor- und Nachteile zu schaffen: auf der Angebotsseite, also bei denjenigen, die die Impfstoffe zur Verfügung stellen, und auf der Nachfrageseite, also bei denjenigen, die die Impfstoffe schließlich erhalten.

Aus langjähriger Forschung ist bekannt, wie man gut über Impfen informieren kann [11]. Klar ist, wann Menschen Impfstoffe mehr und wann weniger wollen. Zum Beispiel haben wir eine geringere Impfbereitschaft, wenn Impfungen weniger effektiv sind. Wäre ein Impfstoff - hypothetisch gesprochen - zu $100 \%$ effektiv, wollen ihn relativ viele in Anspruch nehmen. Auch die Empfehlungsbereitschaft von Gesundheitspersonal richtet sich daran aus. Ein Impfstoff mit einer mittelmäßigen Effektivität von $55 \%$ - ein für Influenza-Impfstoffe nicht unwahrscheinlicher Wert - wird weniger gewollt und empfohlen. Bekannt ist ferner, dass Menschen eine Impfung eher wollen, wenn sie wissen, dass sie damit auch andere schützen können, ein Impfstoff also Herdenimmunität bietet [9]. Derzeit scheint noch nicht sicher, ob die Impfstoffe, die gerade im Rennen sind, das am Ende bieten werden. Das heißt, darauf kann im Moment keine Kommunikation aufgebaut werden. Problematisch wird es, wenn beides zusammenkommt: wenn eine Impfung wenig effektiv ist, aber eigentlich Herdenschutz bieten könnte. Vor dem eben skizzierten Hintergrund bietet eine geringe Effektivität weniger Herdenschutz und Menschen möchten sich diese Impfungen weniger verabreichen lassen. Dies ist allerdings eine Milchmädchenrechnung, da eine wenig effektive Impfung auch keinen umfassenden Herdenschutz bietet, sodass es noch wichtiger ist, sich selbst zu schützen. Dieser Zusammenhang wird nur unbefriedigend verstanden und hat negative Auswirkungen auf die Impfbereitschaft. Hier besteht also intensiver Kommunikationsbedarf - und erstmal Bedarf an Wissen, das es über die neuen COVID-19 Impfstoffe so noch nicht gibt.

Impfen wird als sozialer Vertrag wahrgenommen [8]. Das heißt, jemand lässt sich impfen und schützt damit den anderen - und erwartet dann auch, dass dieser sich impft, um mich mit zu schützen. Dafür ist es notwendig, dass sich eine Mehrheit beteiligt. Daher wird es wichtig sein, von Anfang an die Impfbereitschaft 
des medizinischen Personals zu betrachten, da sie auch die Impfbereitschaft der Bevölkerung beeinflussen könnte.

Impfpflicht. Eine Impfpflicht wäre ein möglicher Ausweg, wird im Moment aber eher abgelehnt. Dennoch beschäftigen wir uns damit, da zum Beispiel bei der Masernimpfung von der Politik dieses ,letzte Mittel“ gewählt wurde, um Impflücken zu schließen. Deswegen untersuchen wir immer wieder, was Pflichtimpfungen bewirken. Und aus meiner Sicht kann nur davor gewarnt werden, eine Impfpflicht überhaupt ausführlich ins Gespräch zu bringen, da erst sehr wenig über diese Impfung, insbesondere zu deren Sicherheit bekannt ist [12]. Eine teilweise Impfpflicht kann sich außerdem negativ auf andere freiwillige Impfungen auswirken: Besonders, wer Impfungen ablehnt, ärgert sich durch die Impfpflicht und lässt tendenziell andere freiwillige Impfungen aus; dies konnte im Zusammenhang mit einer hypothetischen COVID-19 Impfpflicht schon für die Influenzaimpfung gezeigt werden [13-15]. Eine Impfpflicht könnte also ein gut funktionierendes, auf Empfehlungen basierendes Impfsystem bedrohen, wenn die freiwilligen Impfungen - sozusagen aus Trotz - ausgelassen werden.

\section{Fazit}

Zusammengefasst heißt das, dass für die Einführung der COVID-19 Impfungen diverse Herausforderungen bestehen: skeptisches medizinisches Personal, eine sich andeutende Skepsis gegenüber einem genbasierten Impfstoff, eine allgemein eher mittelmäßige Impfbereitschaft gegen COVID-19 sowie sehr viele Unsicherheiten über Impfstoffeigenschaften. Einige Maßnahmen, wie zum Beispiel die Impfpflicht, können mit deutlichen Nebenwirkungen psychologischer Art einhergehen. Für die Akzeptanz der neuen Impfung könnte als Denkmodell die Grippeimpfung herangezogen werden - und zwar mit der Wahrnehmung, dass es sich dabei schon um kein besonderes Erfolgsmodell handelt. Es ist eine Impfung, die ihre Tücken hat und nicht besonders gut angenommen wird: Nicht sehr viele Menschen nutzen diese Impfung [16]. Es besteht für COVID-19 außerdem ein sehr hohes Informationsbedürfnis - das ist gleichzeitig gut und schlecht und vor allem am Ende sehr aufwendig. Es zeigt sich zudem, dass auch Impfgegner nicht faul sind. Schon kursieren Gerüchte über die Wirkungen genbasierter Impfstoffe. Es wird über die Sicherheit debattiert. Daher ist es sehr wichtig, dass transparent und rechtzeitig informiert wird, um sozusagen vor der Welle der möglichen Falschinformationen präsent zu sein und die Szenerie und die Themen selbst zu bestimmen. Bedeutsam scheint auch, entsprechende Informationen nicht einfach unter das Volk zu bringen, sondern eine angemessene Risikokommunikation zu realisieren und den Menschen zuzutrauen, selbst zu entscheiden, ob und wie sie diese Impfung wollen [11].

Bedeutsam erscheint auch der Abbau praktischer Barrieren. Das war vor COVID19 schon wichtig, jetzt ist es so bedeutend wie noch nie, denn: Wer wenig Vertrauen hat, lässt sich auch eher durch äußere Umstände abhalten. Die Diskussion sollte gut gesteuert werden und mit besonderem Augenmerk auf medizinisches Personal jetzt in die nächsten Phasen übergehen. Auch sollten die gesetzlichen Rahmenbedingungen 
verbessert werden, indem auch Befunde, wie sie hier vorgestellt wurden, mit in die Kommunikation oder gar in die Gestaltung von politischen Rahmenbedingungen einbezogen werden.

\section{Literatur}

1. Chen, R.T.: Vaccine risks: real, perceived and unknown. Vaccine 17(Suppl 3), 41-46 (1999)

2. Betsch, C., Wieler, L.H., Habersaat, K.: Monitoring behavioural insights related to COVID19. Lancet 395(10232), 1255-1256 (2020)

3. COSMO group. Corona-Monitor. www.corona-monitor.de

4. Brewer, N.T., Chapman, G.B., Gibbons, F.X., Gerrard, M., McCaul, K.D., Weinstein, N.D.: Meta-analysis of the relationship between risk perception and health behavior: the example of vaccination. Health Psychol. 26(2), 136 (2007)

5. Schmid-Küpke, N. et al.: Cancelled routine vaccination appointments due to COVID-19 pandemic in Germany (2021)

6. Neumann-Böhme, S., et al.: Once we have it, will we use it? A European survey on willingness to be vaccinated against COVID-19. Eur. J. Health Econ. 21(7), 977-982 (2020). https://doi.org/10.1007/s10198-020-01208-6

7. Betsch, C., Schmid, P., Heinemeier, D., Korn, L., Holtmann, C., Böhm, R.: Beyond confidence: development of a measure assessing the $5 \mathrm{C}$ psychological antecedents of vaccination. PLOS ONE eingereicht (2021)

8. Korn, L., Böhm, R., Meier, N. W., Betsch, C.: Vaccination as a social contract. Proc. Nat. Acad. Sci. U.S.A., im Druck (2021)

9. Betsch, C., Böhm, R., Korn, L., Holtmann, C.: On the benefits of explaining herd immunity in vaccine advocacy. Nat. Hum. Behav. 1(3), 0056 (2017)

10. Neufeind, J., Betsch, C., Habersaat, K.B., Eckardt, M., Schmid, P., Wichmann, O.: Barriers and drivers to adult vaccination among family physicians - insights for tailoring the immunization program in Germany. Vaccine 38(27), 4252-4226 (2020)

11. Lewandowsky, S., Cook, J., Schmid, P., Holford, D. L., Finn, A., et al. The COVID-Vaccine Communication Handbook. A practical guide for improving vaccine communication and fighting misinformation. SciBeh. https://hackmd.io/@scibehC19vax/home (2021). Zugegriffem: 18. Febr 2021

12. Omer, S.B., Betsch, C., Leask, J.: Mandate vaccination with care. Nature 571(7766), 469472 (2019)

13. Meier, N.W., Böhm, R., Korn, L., Betsch, C.: Individual preferences for voluntary vs. mandatory vaccination policies: an experimental analysis. Eur. J. Public Health 30(1), 50-55 (2020).

14. Sprengholz, P., Betsch, C.: Herd immunity communication counters detrimental effects of selective vaccination mandates: Experimental evidence. EClinicalMedicine 22, 100352 (2020)

15. Betsch, C., Böhm, R: Detrimental effects of introducing partial compulsory vaccination: experimental evidence. Eur. J. Public Health 26(3), 378-381 (2016)

16. Schmid, P., Rauber, D., Betsch, C., Lidolt, G., Denker, M-L.: (2017) Barriers of influenza vaccination intention and behavior - a systematic review of influenza vaccine hesitancy, 2005-2016. PLOS ONE 12(1), e0170550 (2017). 
Open Access Dieses Kapitel wird unter der Creative Commons Namensnennung - Nicht kommerziell - Keine Bearbeitung 4.0 International Lizenz (http://creativecommons.org/ licenses/by-nc-nd/4.0/deed.de) veröffentlicht, welche die nicht-kommerzielle Nutzung, Vervielfältigung, Verbreitung und Wiedergabe in jeglichem Medium und Format erlaubt, sofern Sie den/die ursprünglichen Autor(en) und die Quelle ordnungsgemäß nennen, einen Link zur Creative Commons Lizenz beifügen und angeben, ob Änderungen vorgenommen wurden. Die Lizenz gibt Ihnen nicht das Recht, bearbeitete oder sonst wie umgestaltete Fassungen dieses Werkes zu verbreiten oder öffentlich wiederzugeben.

Die in diesem Kapitel enthaltenen Bilder und sonstiges Drittmaterial unterliegen ebenfalls der genannten Creative Commons Lizenz, sofern sich aus der Abbildungslegende nichts anderes ergibt. Sofern das betreffende Material nicht unter der genannten Creative Commons Lizenz steht und die betreffende Handlung nicht nach gesetzlichen Vorschriften erlaubt ist, ist auch für die oben aufgeführten nicht-kommerziellen Weiterverwendungen des Materials die Einwilligung des jeweiligen Rechteinhabers einzuholen. 